\title{
Low-Dose High-Pitch Cardiac CT to Evaluate Abnormal Pacemaker Lead Position
}

\author{
Baosheng Li ${ }^{1}$, Chongfu Jia ${ }^{2 *}$ \\ ${ }^{1}$ Department of Radiology, The Affiliated Hospital of Youjiang Medical University for Nationalities, Baise, China \\ ${ }^{2}$ Department of Cardiovascular Radiology, The First Affiliated Hospital of Dalian Medical University, Dalian, China \\ Email: *chongfujia@sina.com
}

How to cite this paper: Li, B.S. and Jia, C.F. (2021) Low-Dose High-Pitch Cardiac CT to Evaluate Abnormal Pacemaker Lead Position. World Journal of Cardiovascular Diseases, 11, 434-438.

https://doi.org/10.4236/wjcd.2021.119040

Received: August 4, 2021

Accepted: September 11, 2021

Published: September 14, 2021

Copyright $\odot 2021$ by author(s) and Scientific Research Publishing Inc. This work is licensed under the Creative Commons Attribution International License (CC BY 4.0).

http://creativecommons.org/licenses/by/4.0/

\begin{abstract}
Displacement of a cardiac pacemaker lead or myocardial perforation by a lead is an infrequent complication of pacemaker implantation. While standard cardiac CT is useful for diagnosing such complications when routine examinations can not, the potential risks associated with exposure to the high doses of radiation used in CT are of concern. Here, we report three cases in which pacemaker lead displacement or myocardial perforation was definitively diagnosed by low-dose cardiac CT used in the high-pitch spiral mode, when other more routine examinations failed to suggest their occurrence. The mean dose of radiation used for definitively diagnosing the three patients was relatively low $(2.19 \mathrm{mSv})$.
\end{abstract}

\section{Keywords}

X-Ray, Computed Tomography, Pacemaker, Lead, Low-Dose

\section{Introduction}

Several case reports have reported pacemaker lead displacement or perforation can be diagnosed by cardiac CT using the standard mode when the routine examinations are nondiagnostic [1] [2] [3]. However, the potential risks due to exposure to higher ionizing radiation associated with standard cardiac CT have raised concerns. The advent of second-generation, dual-source CT has led to the development of a prospective-ECG triggered high-pitch spiral mode for cardiac CT imaging; this mode has demonstrated high and stable image quality, high diagnostic accuracy, and significant dose reductions. Here, we reported three abnormal pacemaker lead cases diagnosed by cardiac CT using this low-dose mode. 


\section{Patient 1}

A 76-year-old man underwent placement of a dual chamber permanent pacemaker for sick sinus syndrome and paroxysmal atrial fibrillation 7 years ago. He complained of pericardial pain and was found poor sensing and pacing parameters on his atrial lead this week, but there was no significant evidence to confirm the reason according to chest radiography and echocardiography. On suspicion of pacemaker lead displacement or perforation, the patient underwent cardiac CT using prospective-ECG triggered high-pitch spiral mode, and revealed the right atrial lead shifted inwardly and perforated through the inner wall of right atrial, adjoining the interior wall of right pulmonary artery (Figure 1(a), Figure (b)). The effective radiation dose was $2.1 \mathrm{mSv}$.

The right atrial lead was removed and replaced by a new one, the chest pain was gone and the sensing and pacing parameters went back to normal, and 1 year later, he was well and was found normal sensing and pacing parameters on his atrial lead too.

\section{Patient 2}

A 56-year-old woman underwent placement of a dual chamber permanent pacemaker for third degree atrioventricular block and syncope 6 years ago. She complained of chest tightness, shortness of breath and weakness these days, and was found poor sensing and pacing parameters on the right atrial lead. The D-dimer was $876 \mathrm{ug} / \mathrm{L}$, and echocardiography revealed there was a small amount of thrombus around the right atrial lead. CT pulmonary angiography using prospective-ECG triggered high-pitch spiral mode was obtained to exclude pulmonary embolism, it demonstrated the right atrial lead shifted down relatively from the top wall, and the distance of the displacement was $6.3 \mathrm{~mm}$. It also showed the thrombus around the right atrial lead. Furthermore, CT revealed drainage of the azygos vein into the right pulmonary vein incidentally and there was no pulmonary embolism (Figures $2(\mathrm{a})-(\mathrm{c})$ ). The effective radiation dose was $2.4 \mathrm{mSv}$.

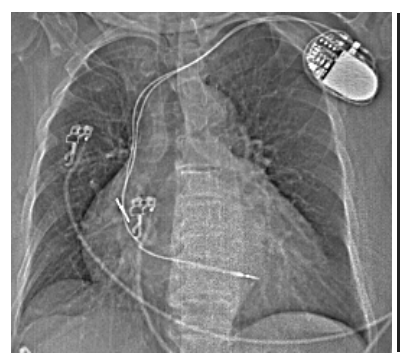

(a)

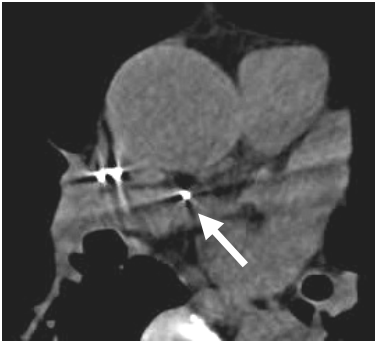

(b)

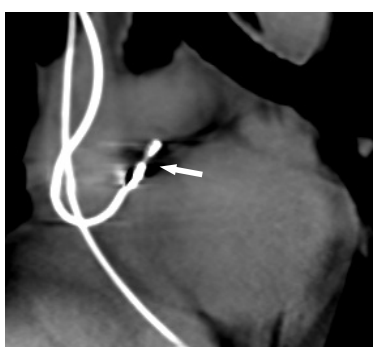

(c)

Figure 1. The chest radiography and CT images from Case 1. The chest radiography (a) showed no significant sign of pacemaker lead perforation. Axial (b) and multiplanar reconstruction. (c) CT images showed the right atrial lead shifted inwardly and perforated through the inner wall of right atrial, adjoining the interior wall of right pulmonary artery (arrows). 


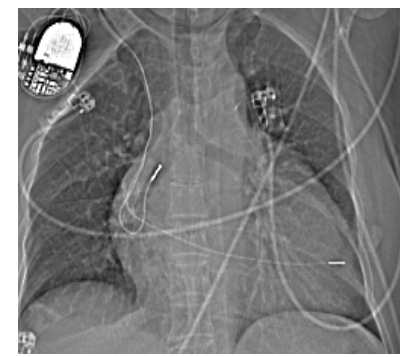

(a)



(c)

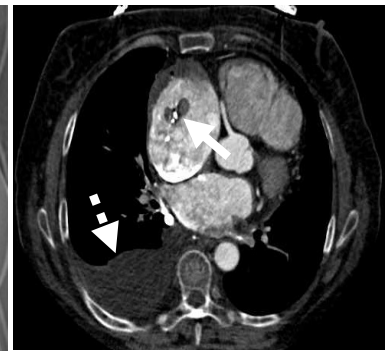

(b)

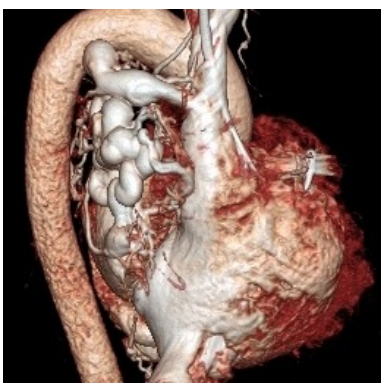

(d)

Figure 2. The chest radiography and CT images from Case 2. The chest radiography (a) showed no significant sign of pacemaker lead perforation. Axial CT image (b) showed the thrombus around the right atrial lead (short arrow) and the right pleural effusion (long arrow). Multiplanar reconstruction CT image (c) showed the right atrial lead shifted down relatively from the top wall (arrow). Volume rendering CT image (d) showed drainage of the azygos vein into the right pulmonary vein.

The patient was treated with warfarin for the thrombus around the right atrial lead, and 2 months later, the thrombus disappeared. The right atrial lead was removed and replaced by a new one, the symptoms were gone and the sensing and pacing parameters went back to normal.

\section{Patient 3}

A 75-year-old woman underwent placement of a dual chamber permanent pacemaker for sick sinus syndrome with swirl and amaurosis 4 years ago. One week ago, the patient came to the hospital for weakness, swirl and chest pain. The subsequent evaluation revealed high pacing threshold and impedance on her right ventricular lead, however the chest radiography and echocardiography showed no signs of lead displacement. The cardiac CT using the prospective-ECG triggered high-pitch spiral mode was performed to exclude coronary heart disease, and it revealed the right ventricular lead deviated from ventricular septum slightly, the distance of displacement was $1.8 \mathrm{~mm}$. Unfortunately, we found she had pulmonary embolism unexpectedly (involving multiple basilar segmental arteries of the bilateral lower lobes) (Figure 3). The effective radiation dose was $2.08 \mathrm{mSv}$.

Similar to case 2, the patient in case 3 received warfarin for treating the pulmonary embolism, and the displaced lead would be removed following complete resolution of the thrombus. 


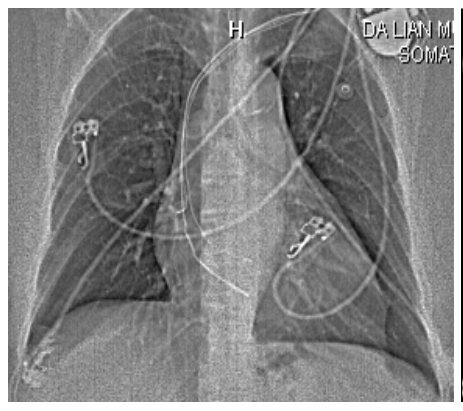

(a)

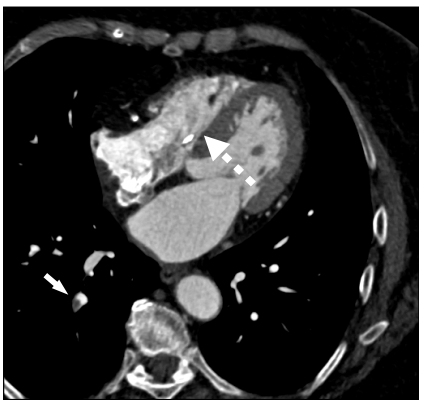

(b)

Figure 3. The chest radiography and CT image from Case 3. The chest radiography (a) showed no significant signs of pacemaker lead displacement. Axial CT image (b) shows the ventricular lead deviated from ventricular septum slightly (long arrow) and the filling defect (short arrow) in the posterior basilar segmental artery of the right lower lobe.

\section{Discussion}

In our report, we diagnosed two leads displacement and one lead perforation of pacemaker according to the low-dose high-pitch cardiac CT and the mean effective radiation dose of these three cases was as low as $2.19 \mathrm{mSv}$. However, all these three abnormal leads were not diagnosed by the chest radiography and echocardiography.

The potential risks associated with exposure to the high doses of radiation used in CT are of concern and in accordance with the "as low as reasonably achievable (ALARA)" principle. The ongoing innovations in CT technology and improvements in CT scanners have led to the introduction of various new high-end CT devices into the clinical sphere. This includes the wide-detector $\mathrm{CT}$, with development of corresponding CT protocols. The radiation dose can be effectively reduced by techniques such as high-pitch spiral mode, low tube voltage, iterative reconstruction, and low tube current. In our three patients, the mean dose of cardiac CT used in the high-pitch spiral mode was relatively low (2.19 mSv).

Lead displacement or perforation after the pacemaker implantation is an infrequent complication. The routine examinations such as chest radiography, echocardiography and fluoroscopy have low sensitivity and accuracy, particularly when the lead is displaced slightly or perforated without displacement. In our three abnormal pacemaker leads, the chest radiography and echocardiography showed no signs of lead displacement or perforation; however the axial CT imaging combining a variety of reconstruction techniques such as maximum intensity projection, curve planar reconstruction and volume rendering can reveal them clearly; particularly, the lead of patient 3 displaced only $1.8 \mathrm{~mm}$, and CT can give a definite diagnosis. Yavari et al. [2] also present the case illustrating the use of CT in a case of right ventricular lead perforation manifesting 5 days after cardiac resynchronization therapy pacing. In addition, we found one patient with pulmonary embolism and one with vascular malformations unexpectedly 
using a single imaging modality of the low-dose cardiac CT.

In summary, the low-dose high-pitch cardiac CT can be a useful adjunct to other routine imaging modalities in the evaluation of possible lead perforation or displacement with significant dose reduction compared to the standard cardiac CT. In addition, radiologists should routinely analyze whether there are other cardiovascular diseases, and provide reliable information for further clinical management.

\section{Conflicts of Interest}

The authors declare no conflicts of interest regarding the publication of this paper.

\section{References}

[1] Gianni, C., Canby, R.C., Anannab, A., et al. (2020) The Subtle Journey of a Right Atrial Lead Hemopneumothorax Due to Subacute Pacemaker Lead Perforation. JACC: Case Reports, 2, 902-906. https://doi.org/10.1016/j.jaccas.2020.05.018

[2] Yavari, A., Khawaja, Z.O., Krishnamoorthy, S. and McWilliams, E.T. (2009) Perforation of Right Ventricular Free Wall by Pacemaker Lead Detected by Multidetector Computed Tomography. EuropAce, 11, 252-254. https://doi.org/10.1093/europace/eun381

[3] Steiner, R.M., Tegtmeyer, C.J., Morse, D., Moses, M.L., Goodman, L.R., Nanda, N., Ravin, C.E., Parsonnet, V. and Flicker, S. (1986) The Radiology of Cardiac Pacemakers. Radiographics, 6, 373-399.

https://doi.org/10.1148/radiographics.6.3.3317544 